\title{
Randomised trial of umbilical arterial catheter position: Doppler ultrasound findings
}

\author{
S T Kempley, H R Gamsu
}

\begin{abstract}
Umbilical arterial catheters (UAC) were randomly assigned in 69 infants to a high $(n=36)$ or to a low $(n=33)$ position. Serial Doppler ultrasound measurements of blood flow velocity in their superior mesenteric arteries, coeliac axis, renal arteries, and anterior cerebral arteries were then obtained.

There were no differences in blood flow velocity between high and low UAC groups on days 1,3 , and 7 . At 2 weeks, those infants with a high UAC still in place had significantly higher velocities in the mesenteric artery than those infants who had no catheter in place. Infants with high UACs remaining in place for more than 7 days were found to have an increase in abdominal distension and tenderness, whereas this was not the case for those with low UACs.

Catheter position has no effect on visceral blood flow if the UAC stays in place for one week or less, whereas prolonged use of a high UAC may alter intestinal blood flow and increase the incidence of abdominal symptoms.
\end{abstract}

There has been a longstanding controversy concerning the role of umbilical arterial catheters (UAC) in the aetiology of necrotising enterocolitis. Case-control studies have produced conflicting results, ${ }^{1-12}$ with the suggestion that UACs may have an independent aetiological role in some birthweight groups while their use is merely associated with other definitive risk factors in others. ${ }^{12}$ If it is assumed that UACs do have a truly causative role, by compromising blood flow in the mesenteric arteries, then catheters placed so that the tip lies above the origin of these arteries (high UACs) would be expected to cause more necrotising enterocolitis than catheters in a low position. However, randomised studies have failed to find any excess of necrotising enterocolitis in those infants with high catheters, ${ }^{13}{ }^{14}$ a finding confirmed in a large randomised study that we have conducted (ST Kempley, S Bennett, BG Loftus, D Cooper, HR Gamsu, unpublished observations).

This is surprising in view of the fact that catheter associated thrombosis has been found in a high proportion of infants with a UAC, 1314 and clinical involvement of the mesenteric arteries was documented principally with high UACs. However, the majority of aortic thrombi are clinically silent and it is possible that catheters, or catheter associated thrombosis, could affect intestinal blood flow without necessarily causing necrotising enterocolitis.

We have therefore used Doppler ultrasound to document the effects of UAC position on blood flow velocity in the superior mesenteric artery (SMA), coeliac axis, and left renal artery. These major branches of the abdominal aorta originate proximally to the tip of UACs placed in a low position, but distal to the tip of UACs placed high; blood flow in them should therefore only be influenced in the latter. We have also included measurements on the anterior cerebral artery (ACA) as an index of each infant's general cardiovascular status.

\section{Methods}

Over a two year period 147 infants admitted to our unit and requiring umbilical arterial catheterisation had their UAC randomly allocated to a high position (T6-T10) or a low position (below L3). Catheters were inserted at a median age of 2 hours to a distance determined from the shoulder-umbilicus length and the position was adjusted after a plain radiograph. The catheters were either of a plain end hole type (Argyle 3.5 French gauge (FG) or Argyle 5.0 FG, Sherwood Medical) or with a side hole and an oxygen sensing electrode at the tip (Neocath 4.0 FG or Neocath 5.0 FG, Biomedical Sensors Ltd). The initial infusate was $5 \%$ dextrose with 1 $\mathrm{IU} / \mathrm{ml}$ heparin, run at $1 \mathrm{ml} / \mathrm{hour}$ in all infants. Blood pressure monitoring was performed via the UAC in all infants.

A birthweight stratified randomisation was used, with permuted blocks within strata, to help produce well matched groups. One hundred and thirty one infants had their catheter successfully inserted into the correct, randomised position. (Over this time period 36 infants had a UAC in place but were not randomised; most of these already had a UAC at the time of transfer from another hospital, or had been considered clinically unsuitable for randomisation before entering the trial.) In order to be included in the study infants were required to have a UAC placed in the position to which they had been randomised and to have had Doppler measurements within the first 24 hours of life.. By excluding data from infants who had their first measurement after 24 hours we ensured that infants were not selected for study on the basis of their later clinical course, and so avoided biased groups. The final study group consisted of 69 infants, 
with 36 high and 33 low UACs; these infants did not differ significantly in any respect from the group who were randomised but not measured. The planned total study size had been 70 subjects, in order to give an $80 \%$ power to detect a $30 \%$ difference in blood flow velocity at a significance level of $5 \%$.

Doppler measurements were performed at 1 day (0-24 hours), 3 days (48-96 hours), and 1 week (120-216 hours) of age, in those infants who were stable enough to tolerate scanning. Measurements were made at 2 weeks (240-316 hours) in most infants who still had a catheter in place, and in some other infants who were still in intensive care. From the original group of 69 who were all measured on day $1,75 \%$ were measured at 3 days, $68 \%$ at 1 week, but only $29 \%$ at 2 weeks. In addition, we made serial measurements on a small number of babies whose attempted UAC placement had failed.

On each occasion we attempted to obtain measurements from the SMA, the coeliac axis, the left renal artery, and the ACA as previously described. ${ }^{15}$ For each artery our readings consisted of a measure of blood flow velocity (the time averaged mean velocity of the peak velocity envelope, corrected for angle of insonation), and a measure of the pulsatility of the velocity waveform (the pulsatility index of Gosling and King). We chose a cerebral artery as the index artery because it would not be affected by high or low UACs, and its measurement could be combined with a clinical imaging scan performed after abdominal Doppler measurements. We found that attempting to combine abdominal Doppler with cardiac output measurements resulted in excessive disturbance to infants who were often small and needing intensive support, and so we did not consider this a suitable measurement for our study.

In our first analysis we assessed the Doppler data according to the randomised position of the UAC (regardless of whether the UAC was still in place at the time of measurement). Subsequently we looked at the influence of actual catheter position at the time of measurement (high, low, or no catheter). In this analysis the 'no catheter' group included infants whose UAC had been removed and

Table 1 Clinical details of the high and low catheter groups who also had Doppler measurements within the first 24 hours of life. (Quantitative measures are medians and ranges)

\begin{tabular}{lcc}
\hline & $\begin{array}{l}\text { High catheter } \\
(n=36)\end{array}$ & $\begin{array}{c}\text { Low catheter } \\
(n=33)\end{array}$ \\
\hline Birth weight (g) & $1160(540-5180)$ & $1400(430-3480)$ \\
Gestation (weeks) & $29(24-40)$ & $30(23-42)$ \\
Small for gestational age & 7 & 7 \\
Caesarean delivery & 15 & 20 \\
Apgar score: & 15 & $27 *$ \\
$\quad<7$ at 1 minute & 10 & 10 \\
<7 at 5 minutes & 16 & 17 \\
Respiratory distress syndrome & 10 & 8 \\
+Extreme prematurity & 6 & 5 \\
Interstitial emphysema & 9 & 5 \\
Infection & 3 & 4 \\
Transient tachypnoea & 0 & 3 \\
Aspiration & 32 & $22(0-41)$ \\
Mechanical ventilation & $20(0-42)$ & \\
$\quad$ Highest peak inspiratory pressure $\left(\mathrm{cm} \mathrm{H}_{2} 0\right)$ & \\
\hline
\end{tabular}

Significant differences: $*=p<0.01$ those infants with a failed catheter placement who nevertheless were studied from day 1.

The data were analysed for the group of infants studied from day 1 until 2 weeks of age. They were divided into a cohort who still had a high catheter in place at 2 weeks, and a cohort who had no catheter in place at 2 weeks. We expressed the results for these two cohorts as the measurement of SMA blood flow velocity divided by ACA blood flow velocity to give the SMA:ACA ratio, in an attempt to control for general rather than specific differences in blood flow velocity between the groups.

The means and $95 \%$ confidence intervals of all the Doppler velocity measurements are presented. We had previously examined Doppler measurements obtained on the first day of life from appropriately grown infants who survived without abdominal symptoms. Velocity data from all arteries were normally distributed; for pulsatility index data only renal artery values had a skewed distribution. Unpaired Student's $t$ tests were used to test the significance of differences between groups, but the MannWhitney $U$ test was used to test differences in renal pulsatility index data. (There were no differences in renal artery pulsatility index between any of the groups.) A log rank analysis was used to assess differences in the duration of catheterisation, with elective removals being counted as 'curtailed follow up'.

For all randomised infants we recorded the occurrence of any abdominal symptoms (abdominal distension, abdominal tenderness or rigidity, blood in the faeces, vomiting, or large bile stained nasogastric aspirates). We divided high and low catheter groups into those who had a catheter in place for more, or less, than 7 days. The relative risk of developing abdominal symptoms after prolonged catheterisation was calculated for the high and low catheter groups (risk for $>7$ days divided by risk for $<7$ days).

\section{Results}

The high and low catheter groups were clinically well matched (table 1), the only statistically significant difference between the groups being a greater number of infants with Apgar scores at 1 minute of less than 7 in the low catheter group. The 5 minute Apgar scores were similar in the two groups, indicating that there was no difference in the incidence of clinically significant asphyxia. Similar catheters were used in the two groups (table 2), with slightly more of the high UACs being used for exchange transfusions. Positive catheter tip cultures were obtained from eight high UACs and two low UACs (just failing statistical significance, $p=0 \cdot 06$ ). High UACs were associated with less blanching or cyanosis of the lower limbs $(p<0.001)$, and stayed in place significantly longer than low UACs $(p<0.05)$.

Randomised UAC position had no effect on blood flow velocity in any of the arteries measured (fig 1). The only significant effect of UAC position was a reduction in the pulsatility 
Table 2 Details of catheters used and their function

\begin{tabular}{lcc}
\hline & $\begin{array}{l}\text { High catheter } \\
(n=36)\end{array}$ & $\begin{array}{l}\text { Low catheter } \\
(n=33)\end{array}$ \\
\hline Catheter type: & & \\
3.5 FG Argyle & 4 & 8 \\
5.0 FG Argyle & 2 & 0 \\
4.0 FG Neocath & 26 & 22 \\
5.0 FG Neocath & 4 & 2 \\
Usage: & 5 & 2 \\
$\quad$ Exchange transfusion & 0 & 1 \\
$\quad$ Total parenteral nutrition & $103(4-614)$ & $99(15-709)$ \\
Duration (hours): median (range) & 8 & 10 \\
Emergency removal & 1 & 3 \\
Replacement & 8 & 2 \\
Positive culture from tip & 8 & $35^{*}$ \\
Blanching or cyanosis of & & \\
lower limbs & & \\
\hline
\end{tabular}

Significant differences: $*=p<0.01$

One type of low catheter not recorded.

index of the SMA with high UACs at 2 weeks of age (high: 2.22, low: 3.20, p<0.05). However, by this stage there were only four infants from the low catheter group left in the study, and only one of these had a catheter in place.

Restricting the analysis to infants who still had a catheter in place at the time of measurement yielded no significant differences between high and low catheter groups (fig 2). However, differences were seen between those
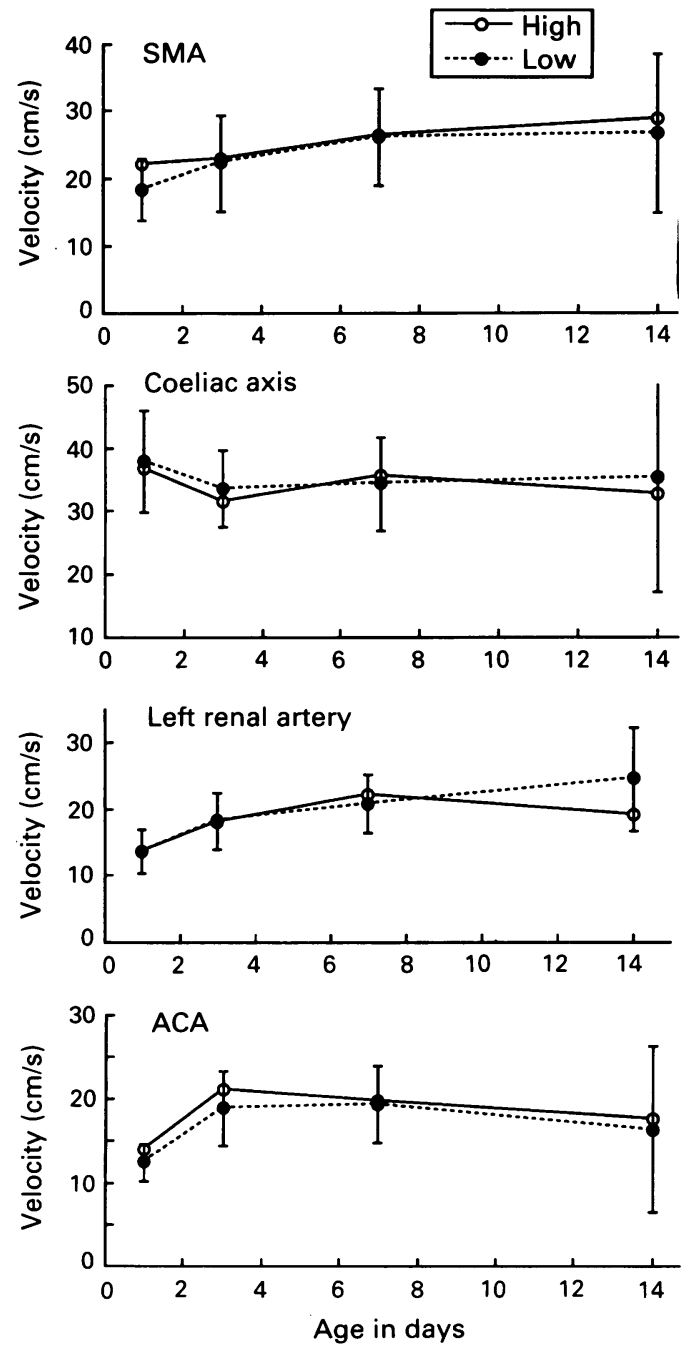

Figure 1 Randomised UAC position and blood flow velocity in the SMA, coeliac axis, left renal artery, and $A C A$. The mean values for high and low UACs are shown, with the $95 \%$ confidence intervals of the difference between the means as error bars on the low group values. infants who had a high catheter in place, and those who had no catheter (those whose catheter had been removed, plus those who had a failed insertion). For the first week of life infants with a catheter in place had lower SMA and coeliac axis blood flow velocities than those infants with no catheter. However, at 2 weeks the situation was reversed, with a significantly higher mean SMA velocity in the infants with a high UAC still in place (33 $\mathrm{cm} / \mathrm{s}, \mathrm{n}=6$ ) compared with those infants who had no catheter $(24.7, \mathrm{~cm} / \mathrm{s}, \mathrm{n}=13, \mathrm{p}<0.05)$. ACA and renal artery velocity were consistently slightly higher in the group with no catheter.

Figure 3 shows the SMA:ACA blood flow velocity ratio in those cohorts defined by their UAC status at 2 weeks. A high ratio was apparent in the cohort with a high UAC in place at 2 weeks, but was not present in them at an earlier age. A similar but non-significant trend was seen in the coeliac axis. Infants who
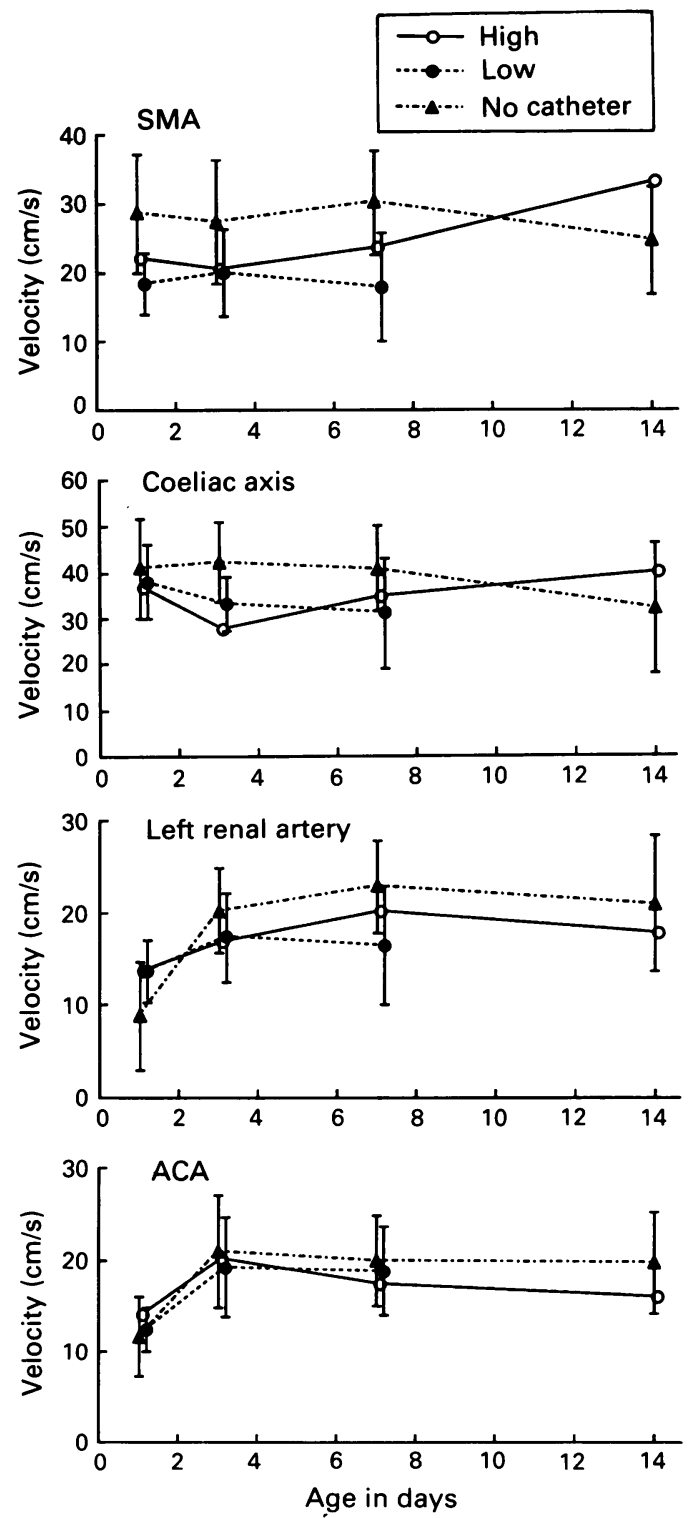

Figure 2 Actual UAC position at the time of measurement, and blood flow velocity in the SMA, coeliac axis, left renal artery, and $A C A$. The means for the three groups are shown. The $95 \%$ confidence intervals of the difference between the high group and the other groups are shown as error bars on the low and no catheter groups. 


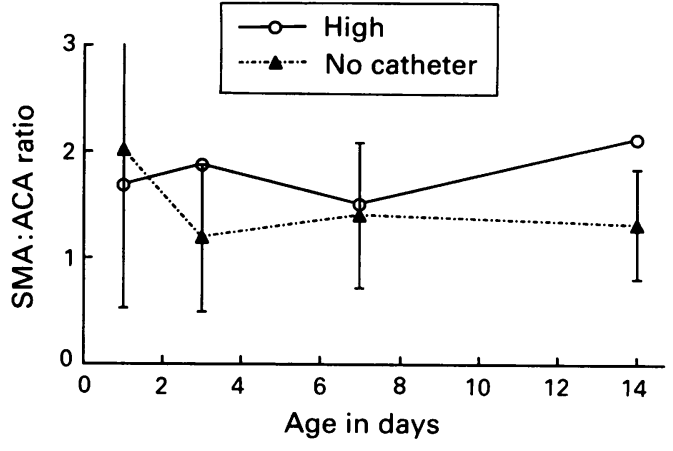

Figure 3 SMA:ACA blood flow velocity ratio. Cohorts followed up from day 1 until 2 weeks and defined by catheter status at 2 weeks. Mean values are shown with the 95\% confidence intervals of the difference between the means.

had a catheter still in place at 2 weeks had a lower mean birth weight (896 g v $1080 \mathrm{~g}$ ), but this difference was not statistically significant. At 2 weeks enteral feeds were being received by three of the six infants with a high UAC in place and $6 / 13$ of those without a UAC. No infant had a clinical patent ductus arteriosus. Mechanical ventilation was still required by all of those with a UAC in place, but by only four of those with no catheter. All the catheters still in place at 2 weeks were of the Neocath type.

Using ultrasound, large aortic thrombi were clearly seen after the removal of the UAC in two infants with high Neocath catheters. One of these infants was asymptomatic, but the other died after a nearly complete occlusion of the SMA and coeliac axis. At the time symptoms developed, this infant had high blood flow velocity in the coeliac axis and the SMA.

Clinical data from all randomised infants showed that in the high catheter group there was an increased risk of abdominal distension, rigidity, and tenderness if the catheter stayed in place for more than a week (table 3). No such excess was seen in the low catheter group. There were no cases of necrotising enterocolitis among infants who had Doppler studies performed, but it did occur in three infants randomised to a high catheter and one randomised to a low catheter.

\section{Discussion}

Even though our study included an adequate number of subjects, we did not find any differences in blood flow velocity in the first week of life between those infants randomised to receive a high or a low UAC. At 2 weeks the only significant difference between the

Table 3 Numbers of infants with abdominal distension, tenderness, or rigidity according to catheter position and the duration of catheterisation. The relative risk and $95 \%$ confidence intervals (CI) of abdominal symptoms that prolonged catheterisation carries is shown for high and low catheter groups

\begin{tabular}{lll}
\hline & High catheter & Low catheter \\
\hline Catheter in 0-7 days & $8 / 47$ & $7 / 47$ \\
Catheter in >7 days & $10 / 22$ & $3 / 15$ \\
Relative risk & $2 \cdot 7$ & $1 \cdot 3$ \\
$(95 \%$ CI) & $(1 \cdot 3-5 \cdot 3)$ & $(0 \cdot 4-4 \cdot 5)$ \\
\hline
\end{tabular}

groups was an increased pulsatility index in the SMA of the high UAC group. As an isolated finding this should be viewed with some caution, as it was the only positive result from 16 tests of significance, and there was only one infant with a low UAC still in place at this stage.

Further analysis of the effects of actual catheter position does suggest that high UACs may alter mesenteric blood flow if they stay in place for 2 weeks or more. Compared with those infants who have no catheter, infants who have a high UAC in situ at 2 weeks have a raised SMA blood flow velocity, with a nonsignificant increase in coeliac axis blood flow velocity. This pattern is the opposite to that seen in the first week, and is not seen until 2 weeks even when we follow up the groups as cohorts. We might have expected those infants with a UAC remaining in place at 2 weeks to have lower intestinal blood flow as they were smaller, a greater number required to be ventilated, and they had a lower ACA blood flow velocity than those whose UAC had been removed. Intrauterine hypoxia, ${ }^{15}$ patent ductus arteriosus, ${ }^{16}$ and enteral feeding ${ }^{17} 18$ have all been shown to alter Doppler measurements of SMA blood flow velocity, but the groups did not differ with respect to the frequency of any of these factors. We therefore believe that the abnormalities we have demonstrated are related to prolonged catheterisation rather than resulting from differences in the subject groups.

High UACs may have been expected to reduce flow in the SMA, so why should their prolonged use result in an increase of SMA blood flow velocity? Since completing this study we have seen high SMA blood flow velocity in a baby with a positive culture of Candida albicans from the tip of the UAC and candida emboli in the mesenteric vessels. Catheter tip infection could produce mesenteric vasodilatation as a result of local bacteraemia, and high UACs may be more prone to colonisation because they are in place for a longer period. Also, emboli or other intermittent ischaemic phenomena resulting from high UACs may have been missed by this study, and such events could eventually produce a reactive hyperaemia following multiple small ischaemic/septic episodes. We have not investigated the effects of sampling from the catheter, which may also predispose to such effects. It is important, however, to point out that high arterial blood flow velocity does not necessarily mean that there is high arterial volume flow. In the one infant with documented coeliac and SMA thrombosis, with hepatic and bowel infarction, arterial blood flow velocity remained high throughout, despite a presumably low absolute volume flow. The high observed velocity might well have resulted from a considerable reduction in vessel diameter, in much the same way that high velocity flow is seen through stenotic cardiac valves. Catheter associated thrombosis may therefore provide the best explanation of the increase of blood flow velocity in the SMA that we demonstrated. 
Are our findings of any clinical significance, except in rare cases of near total mesenteric vessel occlusion? During this study we recorded all abdominal symptoms, and found an increased risk of abdominal distension, tenderness, or rigidity only in those infants who had a high UAC in place for more than one week. We are not able to comment on whether low UACs produce any Doppler abnormalities at 2 weeks, because few were still in place then. The fact that their prolonged use does not produce any increase in abdominal symptoms suggests that blood flow abnormalities did not occur. Nearly all of the catheters that were in place for more than a week were of the Neocath type, with an oxygen sensing electrode in the tip. We were therefore unable to determine whether catheter type had any influence on the incidence of Doppler abnormalities or abdominal symptoms.

We would suggest that prolonged use of high UACs may result in bowel injury without necessarily causing necrotising enterocolitis. Such bowel injury is most likely to be the result of thromboembolism. It is therefore important to differentiate thromboembolic ischaemic necrosis from necrotising enterocolitis. If we become alert to the possibility of this diagnosis in infants with high catheters, the early use of thrombolytic treatment or of surgery could improve the outcome from catheter associated thrombosis.

We would recommend that high UACs should be used initially for all infants who require umbilical catheterisation, as they remain functional for a longer period and cause less lower limb ischaemia than low UACs. Further research is needed to determine the best policy to be followed for the infant who requires catheterisation for more than a week, when high UACs may disturb visceral blood flow and cause bowel injury. It is possible that the use of less thrombogenic materials in the manufacture of catheters may reduce the incidence of thrombosis related problems, but any trials of such materials should study adequate numbers of high catheters which stay in place for more than a week, as this is the group where significant problems occur.

1 Roback SA, Foker J, Frantz IF, Hunt CE, Engel RR, Leonard AS. Necrotizing enterocolitis. Arch Surg 1974; 109:314-9.

2 Frantz ID, L'Heureux PL, Engel RR, Hunt CE. Necrotizing enterocolitis. $\mathcal{F}$ Pediatr 1975;86:259-63.

3 Bunton GL, Durbin GM, McIntosh N, et al. Necrotising enterocolitis. Arch Dis Child 1977;52:772-7.

Yu VYH, Tudehope DI. Neonatal necrotising enterocolitis: perinatal risk factors. Med $\mathcal{F}$ Aust 1977;i:688-93.

5 Lehmiller DJ, Kanto WP. Relationships of mesenteric thromboembolism, oral feeding and necrotizing enterocolitis. F Pediatr 1978;92:96-100

6 Ryder RW, Shelton JD, Guinan ME. Necrotising enterocolitis: a prospective multicenter investigation. $\mathrm{Am} \mathcal{J}$ Epidemiol 1980:112:113-23.

7 Smith MF, Borriello SP, Clayden GS, Casewell MW. Clinical and bacteriological findings in necrotising enterocolitis: a controlled study. F Infect 1980;2:23-31.

8 Stoll BJ, Kanto WP, Glass RI, Nahmias AJ, Brann AW. Epidemiology of necrotising enterocolitis: a case control study. F Pediatr 1980;96:447-51.

9 Kliegman RM, Hack M, Jones P, Fanaroff AA. Epidemiologic study of necrotising enterocolitis among low-birthweight infants. $\mathcal{F}$ Pediatr $1982 ; 100: 440-4$.

10 Han VKM, Sayed H, Chance GW, Brabyn DG, Shaheed W. An outbreak of Clostridium difficile necrotizing enterocolitis: a case for oral vancomycin therapy. Pediatrics 1983;71:935-41.

11 Yu VYH, Joseph R, Bajuk B, Orgill A, Astbury J. Perinatal risk factors for necrotising enterocolitis. Arch Dis Child 1984;59:430-4.

12 Palmer SR, Thomas SJ, Cooke RWI, et al. Birthweight specific risk factors for necrotising enterocolitis. $\mathcal{F}$ Epidemiol Community Health 1987;41:210-4.

13 Mokrohisky ST, Levine RI, Blumhagen JD, Wesenberg RL, Simmons MA. Low positioning of umbilical-artery catheters increases associated complications in newborn infants. $N$ Engl $\mathcal{F}$ Med 1978;299:561-4.

14 Wesstrom G, Finnstrom O, Stenport G. Umbilical artery catheterisation in newborns: thrombus in relation to catheter type and position. Acta Paediatr Scand 1979;68: 575-81.

15 Kempley ST, Gamsu HR, Vyas S, Nicolaides K. Effects of intrauterine growth retardation on postnatal visceral and cerebral blood flow velocity. Arch Dis Child 1991;66: 1115-8.

16 Coombs RC, Morgan MEI, Durbin GM, Booth IW, McNeish AS. Gut blood flow velocities in the newborn: effects of patent ductus arteriosus and parenteral indomethacin. Arch Dis Child 1990;65:1067-71.

17 Leidig E. Doppler analysis of superior mesenteric artery blood flow in preterm infants. Arch Dis Child 1989;64: 476-80.

18 Gladman G, Sims DG, Chiswick ML. Gastrointestinal blood flow velocity after the first feed. Arch Dis Child 1991;66:17-20. 